05,13

\title{
Интерференция магнитоупругих волн в пленке железо-иттриевого граната
}

\author{
(С) С.Л. Высоцкий, А.В. Кожевников, Ю.А. Филимонов \\ Саратовский филиал Института радиотехники и электроники им. В.А. Котельникова РАН, \\ Саратов, Россия \\ E-mail: vysotsı@gmail.com
}

Поступила в Редакцию 9 апреля 2021 г.

В окончательной редакции 9 апреля 2021 г.

Принята к публикации 19 апреля 2021 г.

\begin{abstract}
Экспериментально исследована интерференция встречно распространяющихся в пленке железо-иттриевого граната магнитоупругих волн, возбуждаемых и принимаемых токовыми антеннами. Показано, что с помощью регулировки фазы и амплитуды одной из взаимодействующих волн на частотах вблизи частот магнитоупругих резонансов может быть обеспечена чувствительность уровня сигнала на выходной антенне к изменению величины поля подмагничивания более $250 \mathrm{~dB} /$ Ое. Обнаруженный эффект может быть полезен при разработке датчиков магнитного поля.
\end{abstract}

Ключевые слова: интерференция, магнитоупругие волны, датчик магнитного поля.

DOI: 10.21883/FTT.2021.09.51249.02H

\section{1. Введение}

Интерференция спиновых волн (СВ) активно изучается на протяжении более десятка лет. Экспериментальные исследования и их численное моделирование проводятся с использованием спин-волновых интерферометров типа Маха-Цендера [1-4] или на основе $\Psi$-образных волноводов [5-7], а также решеток из ортогонально пересекающихся волноводов [8-12], в которых возбуждение и прием СВ осуществляется токовыми антеннами. Изменение амплитуды или фазы накачки, поступающей на одну из возбуждающих антенн, позволяет на некоторых фиксированных частотах изменять характер интерференции СВ на приемной антенне с конструктивной на деструктивную (или наоборот) [13,14], что радикально сказывается на амплитуде и фазе СВ на выходе устройств и может быть использовано для разработки логических устройств [1-7], голографической памяти $[8,9,11]$, сигнальных процессоров $[15,16]$ и магнитных сенсоров $[17,18]$. В последнем случае используется тот факт, что в спектре СВ на некоторых частотах чувствительность амплитуды и фазы измеряемого на выходной антенне сигнала к изменению величины поля подмагничивания достигает $200 \mathrm{~dB} / \mathrm{Oe}$ и $10 \pi / \mathrm{Oe}$ соответственно.

Следует отметить, что измерение величины постоянного магнитного поля $H$ с помощью устройств, использующих распространение спиновых волн в ферритовых волноводах, исследуется уже более двух десятков лет. В качестве измеряемых параметров, кроме указанных выше амплитуды и фазы сигнала с выходного преобразователя $\mathrm{CB}$, использовались величина отраженной от входного преобразователя СВ мощности [19], а для магнонных кристаллов - центральная частота полосы непропускания [20-22]. Рассматривались варианты повышения температурной стабильности таких датчиков [23] и их интегрирования в измерительные модули [24]. Однако чувствительность описанных датчиков не превышала $100 \mathrm{~dB} / \mathrm{Oe}$ [19-22].

В настоящей работе рассматриваются эффекты, сопровождающие интерференцию магнитоупругих волн, распространяющихся в волноводе из пленки железоиттриевого граната навстречу друг другу, а также возможность их использования для разработки датчика магнитного поля.

\section{2. Исследуемый образец и методика измерений}

Для проведения измерений использовалась пленка легированного железо-иттриевого граната (ЖИГ) толщиной по паспорту $d \approx 1.9 \mu \mathrm{m}$, намагниченностью насыщения $4 \pi M \approx 1750 \mathrm{G}$, выращенная эпитаксиально на подложке гадолиний-галлиевого граната (ГГГ) с кристаллографической ориентацией (111) толщиной $D=550 \mu \mathrm{m}$. Из пленки был вырезан волновод с плоскостными размерами $4 \times 15 \mathrm{~mm}$, который размещался на трех мирополосковых антеннах $1,2,3$ (см. вставки $a, b$ к рис. 1) шириной $50 \mu \mathrm{m}$, длиной $4.5 \mathrm{~mm}$. Антенны 1 и 2 использовались для возбуждения магнитостатических волн (MCB), антенна 3 являлась выходной. Расстояние между выходной и каждой из входных антенн составляло $3 \mathrm{~mm}$. СВЧ сигнал с векторного анализатора цепей поступал на делитель $S$ и затем на входные антенны, при этом между делителем и одной из антенн включались фазовращатель и регулируемый аттенюатор. Магнитное поле $H$ могло прикладываться в плоскости пленки параллельно антеннам для возбуждения поверхностных МСВ 


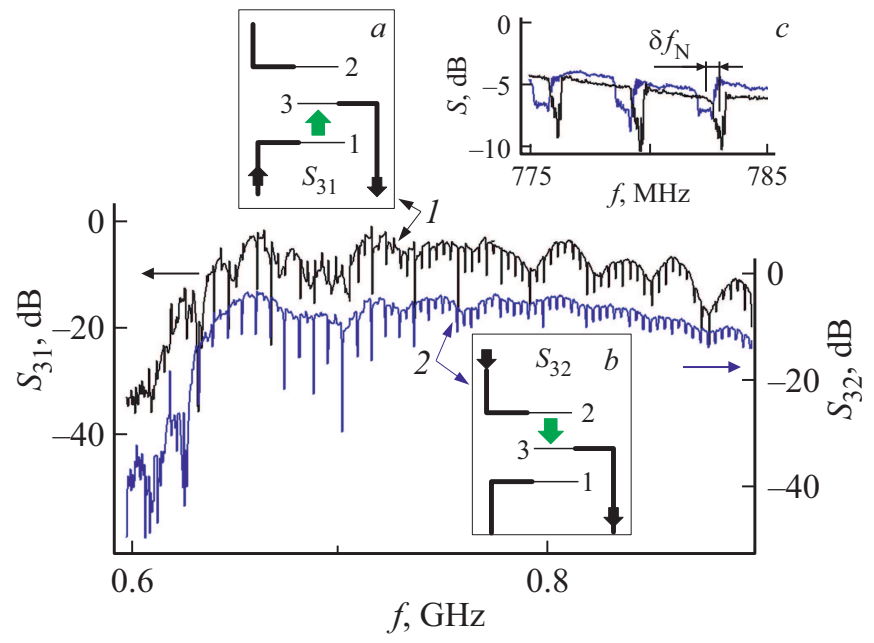

Рис. 1. Частотные зависимости модуля коэффициента передачи ПМСВ с антенны $1 S_{31}$ (кривая 1 ) и антенны $2 S_{32}$ (кривая 2) на антенну 3. $H=29$ Ое.

(ПМСВ) или перпендикулярно плоскости пленки для возбуждения прямых объемных МСВ (ПОМСВ) [25]. Сигнал с выходной антенны перед подачей на вход анализатора цепей усиливался на $27 \mathrm{~dB}$.

На рис. 1 приведены частотные зависимости модуля коэффициента передачи ПМСВ на выходную антенну для случаев возбуждения ПМСВ только антенной 1 $S_{31}(f)$ (см. кривую 1 и вставку $\left.a\right)$ или только антенной $2 S_{32}(f)$ (см. кривую 2 и вставку $b$ ) при $H=29$ Ое. При выбранном уровне выходной мощности анализатора цепей $P=-38 \mathrm{dBm}$ процессы трехмагнонного распада ПМСВ [25] не наблюдались. Видно, что обе зависимости содержат эквидистантно расположенные вблизи частот $f_{N}$ узкополосные участки увеличения потерь ПМСВ („проваль“), при этом для каждой кривой измеренный частотный интервал между центральными частотами соседних провалов $\Delta f_{N, N+1} \approx 3 \mathrm{MHz}$ хорошо соответствовал результатам расчета (в приближении $d \ll D)$ с помощью известного для магнитоупругих волн (МУВ) соотношения [26] $\Delta f_{N, N+1}=V /(2 D)$, где $V=3.57 \cdot 10^{5} \mathrm{~cm} / \mathrm{s}$ - скорость поперечных акустических волн в ГГГ, $N$ - целое число полуволн акустической сдвиговой волны по толщине структуры. Однако абсолютные значения $f_{N}$ на кривых 1 и 2 различались на величину $\delta f_{N} \approx 0.19 \ldots 24 \mathrm{MHz}$. С использованием экспериментально измеренных фазочастотных характеристик $\phi_{31}(f)$ и $\phi_{32}(f)$ с помощью известного соотношения [27] $q(f)=\phi(f) / L$, где $q-$ волновое число, были построены дисперсионные зависимости ПМСВ. При сравнении полученных кривых с результатами расчета при параметрах эксперимента и варьировании толщины пленки ЖИГ оказалось, что совпадение результатов достигается при использовании толщин пленок ЖИГ 1.85 и $2 \mu \mathrm{m}$ для случаев распространения только одной или только другой МУВ. Проведенная оценка разницы частот $f_{N} \approx V_{t} N /[2(D+d)]$, рассчитанных с учетом толщины пленки ЖИГ при $N=240$ (для $f_{N} \approx 776 \mathrm{MHz}$ ) составила $0.21 \mathrm{MHz}$, что хорошо согласуется с экспериментально измеренными величинами $\delta f_{N}$. Таким образом, исследуемая пленка ЖИГ оказалась неоднородной по толщине.

\section{3. Результаты измерений}

При одновременной подаче СВЧ-мощности на обе входные антенны амплитуда и фаза сигнала с выходной антенны определяются как результат интерференции в месте ее расположения встречно распространяющихся поверхностных (МУВ). На рис. 2 кривые 1 и 2 представляют, соответственно, частотные зависимости амплитуды $S(f)$ и фазы $\varphi(f)$ сигнала на выходной антенне при $H=33$ Ое. Видно, что осцилляциям амплитуды на некоторых частотах $f_{N}$ (например, помеченной звездочкой на кривой 1 ) соответствуют на характеристике $\varphi(f)$ скачки фазы, достигающие 360 градусов (помечен
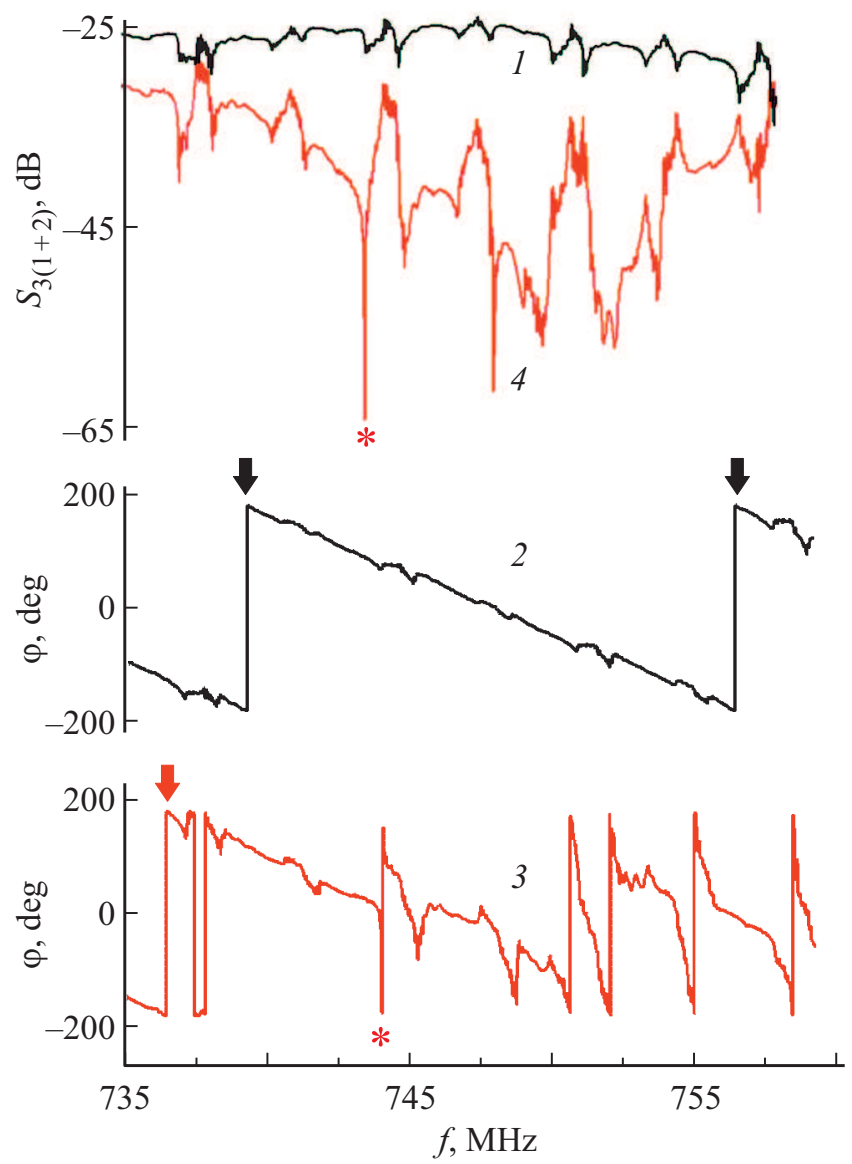

Рис. 2. Частотные зависимости модуля коэффициента передачи ПМСВ $S_{3(1+2)}$ (кривые 1 и 4 ) сигнала на выходной антенне в отсутствие регулировки (кривая 1) и при ее наличии (кривая 4). Кривые 2 и 3 - частотные зависимости фазы сигнала на выходной антенне в отсутствие регулировки (кривая 2) и при ее наличии (кривая 3). $H=29$ Ое. 

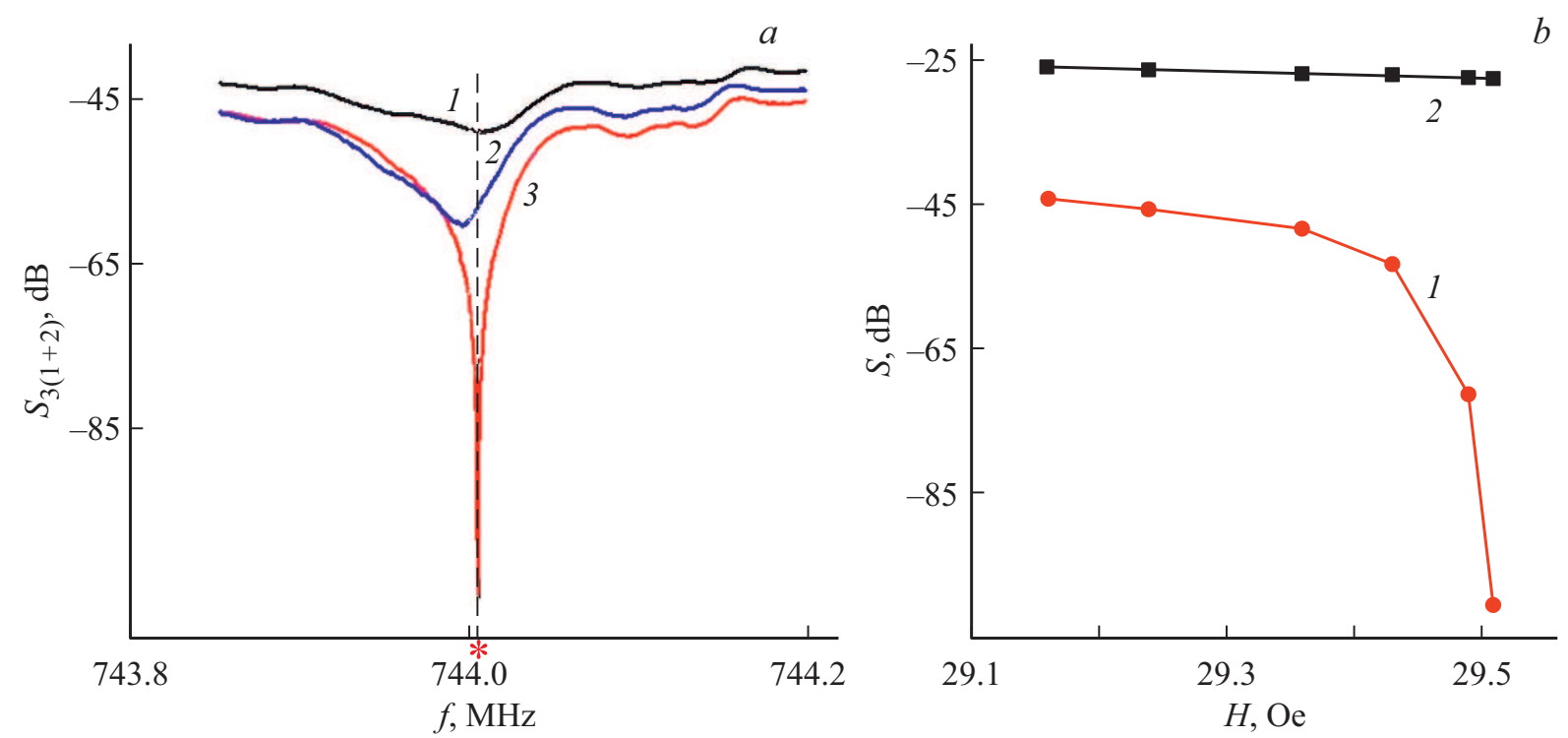

Рис. 3. а) частотные зависимости модуля коэффициента передачи ПМСВ $S_{3(1+2)}$ при $H=29.16$ Ое, 29.43 Ое и 29.51 Ое (кривые 1,2 и 3 соответственно). b) зависимость модуля коэффициента передачи ПМСВ от величины внешнего магнитного поля $H$ на частоте $744.01 \mathrm{MHz}$ в случае распространения двух интерферирующих волн $S_{3(1+2)}$ (кривая 1$)$ и одной ПМСВ $S_{31}$ (кривая 2).
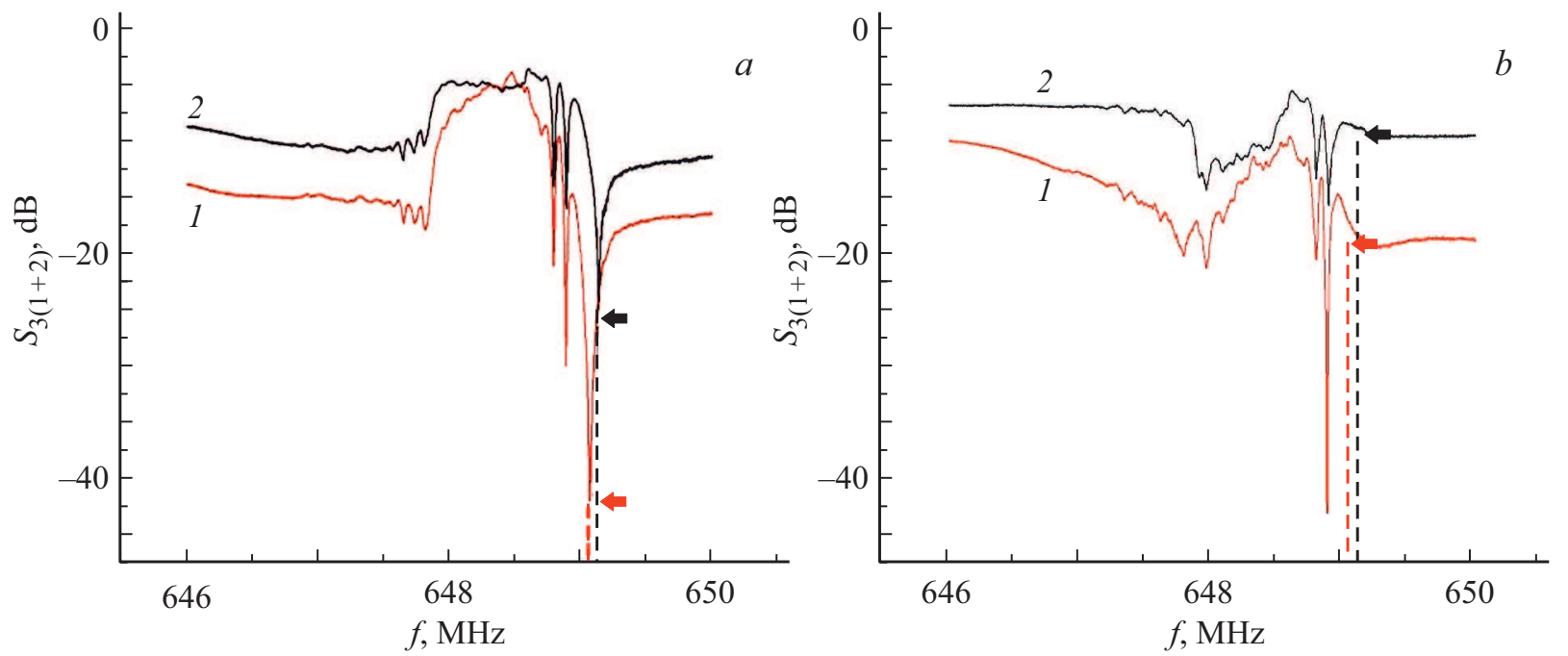

Рис. 4. Экспериментальные (кривые 1,3 ) и рассчитанные (кривые 2,4 ) зависимости модуля коэффициента передачи на антенну 3 для величин $H=28.56 \mathrm{Oe}(a)$ и $H=29.12 \mathrm{Oe}(b)$.

звездочкой на кривой 2, где, кроме того, стрелками показаны осцилляции фазы ПМСВ при кратности ее длины волны и расстояния между антеннами). Такое изменение фазы свидетельствует о смене на этой частоте характера интерференции. При этом с помощью изменения фазы и вносимого ослабления в регулируемом канале можно получить аналогичный результат и на других дискретных частотах. Так, например, кривая 4 на рис. 2 отвечает ситуации, когда в результате противофазного сложения на выходной антенне сигналов ПМСВ вклад в уровень суммарного сигнала в центре рассматриваемой полосы частот обусловлен преимущественно упругой компонен- той магнитоупругой волны. При этом наблюдаются аналогичные описанным выше участки резкого изменения амплитуды (помечены звездочками на кривой 4 рис. 2) и фазы (помечены звездочками на кривой 3 рис. 2) выходного сигнала. Прямоугольником на кривой 3 выделена область, где уровень сигнала на выходной антенне оказывается недостаточным (сравн. с кривой 4) для определения его фазы использованным прибором.

Положение и глубина заграждения участков резкого изменения в частотной зависимости амплитуды сигнала с выходной антенны, возникающих в результате интерференции, зависит, в отличие от частот $f_{N}$, от величины 


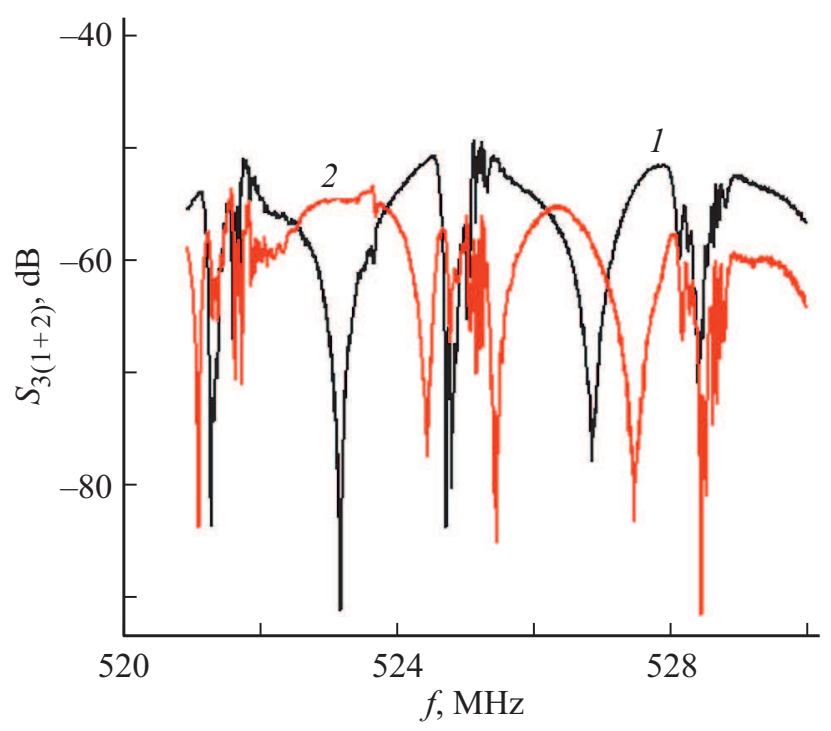

Рис. 5. Частотные зависимости модуля коэффициента передачи ПОМСВ $S_{3(1+2)}$ в отсутствие регулировки (кривая 1 ) и при ее наличии (кривая 2). $H=1.63 \mathrm{kOe}$.

поля подмагничивания, что может быть использовано на практике. На рис. 3, $а$ приведены полученные для исследованной структуры частотные зависимости $S_{3(1+2)}$ для $H=29.16 \mathrm{Oe}, 29.43 \mathrm{Oе}$ и $29.51 \mathrm{Oе}$ (кривые 1,2 и 3 , соответственно). Видно, что на частоте $744.01 \mathrm{MHz}$ изменение уровня выходного сигнала при изменении величины внешнего поля в указанном интервале превышает $40 \mathrm{~dB}$. При этом в зависимости $S_{3(1+2)}(H)$ есть интервал полей $H$, в котором ее крутизна превышает $250 \mathrm{~dB} / \mathrm{Oе} \mathrm{(см.} \mathrm{кривую} 1$ на рис. $2, b$ ), что может быть использовано при разработке высокочувствительных датчиков магнитного поля. Подчеркнем, что в случае одной магнитоупругой волны для этой же частоты изменение величины поля подмагничивания в указанных пределах не приводит к заметному изменению уровня выходного сигнала (см. кривую 2 на рис. $3, b)$.

С использованием измеренных зависимостей модуля и фазы коэффициентов передачи МУВ с антенны 1 на антенну 3 и с антенны 2 на антенну 3 проведено моделирование взаимодействия этих волн в области расположения 3 антенны по правилам сложения комплексных коэффициентов передачи $\dot{S}=S_{31} e^{i \varphi_{31}}+S_{32} e^{i \varphi_{32}}$, где $i=\sqrt{-1}$. На рис. 4 показано сравнение экспериментальных (кривые 1,3) и рассчитанных (кривые 2, 4) зависимостей модуля коэффициента передачи для величин $H=28.56$ Oе (рис. $4, a)$ и $H=29.12$ Ое (рис. $4, b)$. Можно видеть их хорошее качественное совпадение. Из количественного сравнения рис. 4, $a$ и $b$ видно, что в эксперименте на частоте $649.08 \mathrm{MHz}$ (красная штриховая линия) глубина провала (показана красной стрелкой) изменяется при изменении величины $H$ на $23 \mathrm{~dB}$, а в расчете для частоты $649.14 \mathrm{MHz}$ (черная штриховая линия и черные стрелки) это изменение составляет $15 \mathrm{~dB}$.
При нормальном намагничивании пленки ЖИГ, отвечающем возбуждению ПОМСВ, наблюдались аналогичные эффекты, однако уровень потерь ПОМСВ на распространение был на 10-15 dB выше, чем для случая ПМСВ - см. рис. 5.

\section{4. Заключение}

Таким образом, в работе исследовано взаимодействие встречно распространяющихся в пленке ЖИГ поверхностных и прямых объемных МУВ. Показано, что в частотной области наблюдения МСВ с помощью регулировки ослабления и фазы одной из взаимодействующих волн можно на дискретных частотах обеспечить чувствительность уровня сигнала к изменению величины внешнего магнитного поля более $250 \mathrm{~dB} / \mathrm{Oe}$, что может быть применено при разработке высокочувствительных датчиков магнитного поля.

\section{Финансирование работы}

Работа выполнена в рамках государственного задания № 0030-2019-0013 „Спинтроника“ при финансовой поддержке Российского фонда фундаментальных исследований (проект № 20-07-00973).

\section{Конфликт интересов}

Авторы заявляют, что у них нет конфликта интересов.

\section{Список литературы}

[1] M.P. Kostylev, A.A. Serga, T. Schneider, B. Leven. Hillebrands B 87, 153501 (2005).

[2] T. Schneider, A.A. Serga, B. Leven, Hillebrands, R.L. Stamps, M.P. Kostylev. Appl. Phys. Lett. 92, 022505. (2008).

[3] A.V. Chumak, A.A. Serga, B. Hillebrands. Nature Commun. 5, 4700. (2014).

[4] O. Rousseau, B. Rana, R. Anami, M. Yamada, K. Miura, S. Ogawa. Otani Y. Sci. Rep. 5, 09873 (2015).

[5] S. Klingler, P. Pirro, T. Brächer, B. Leven, B. Hillebrands, A.V. Chumak. Appl. Phys. Lett. 105, 152410 (2014).

[6] S. Klingler, P. Pirro, T. Brächer, B. Leven, B. Hillebrands, F.V. Chumak. Appl. Phys. Lett. 106, 212406 (2015).

[7] T. Fischer, M. Kewenig, D.A. Bozhko, A.A. Serga, I.I. Syvorotka, F. Ciubotaru, C. Adelmann, B. Hillebrands, A.A. Chumak. Appl. Phys. Lett. 110, 152401 (2017).

[8] A. Khitun. J. Appl. Phys. 113, 164503 (2013).

[9] F. Gertz, A. Kozhevnikov, Y. Filimonov, A. Khitun. IEEE Trans. Magn. 51, 4, P. 1 (2014).

[10] A. Balynsky, Y. Kozhevnikov, Khivintsev, T. Bhowmick, D. Gutierrez, H. Chiang, G. Dudko, Y. Filimonov, G. Liu. J. Appl. Phys. 121, 024504 (2017).

[11] A. Kozhevnikov, F. Gertz, G. Dudko, Y. Filimonov, A. Khitun. Appl. Phys. Lett. 106, 142409 (2015).

[12] M. Balynskiy, H. Chiang, D. Gutierrez, A. Kozhevnikov, Y. Filimonov, A. Khitun. J. Appl. Phys. 123, 144501 (2018).

[13] A. Khitun, M. Bao, K.L. Wang. J. Phys. D 43, 264005 (2010). 
[14] G. Csaba, Á. Papp, W. Porod. Phys. Lett. A. 381, 1471 (2017).

[15] A. Papp, W. Porod, A.I. Csurgay, G. Csaba. Sci. Rep. 7, 9245 (2017).

[16] G. Csaba, A. Papp, W. Porod. J. Appl. Phys. 115, 17C741 (2014).

[17] M. Balynsky, D. Gutierrez, H. Chiang, A. Kozhevnikov, G. Dudko, Y. Filimonov, A. Balandin, A. Khitun. Sci. Rep. 7, 11539 (2017).

[18] M. Balinskiy, H. Chiang, A. Kozhevnikov, Y. Filimonov, A.A. Balandin, A. Khitun. J. Magn. Magn. Mater. 514, 167046 (2020).

[19] A.O. Kaya, S. Atalay, H. Gencer, O.A. Kaya, V.S. Kolat, T. Izgi. Acta Phys. Polonica A. 127, 4, 937 (2015).

[20] M. Inoue, A. Baryshev, H. Takagi, P.B. Lim, K. Hatafuku, J. Noda, K. Togo. Appl. Phys. Lett. 98, 132511 (2011).

[21] P. Talbot, A. Fessant, J. Gieraltowski. Proc. Eng. 120, 1241 (2015).

[22] R.G. Kryshtal, A.V. Medved. Appl. Phys. Lett. 100, 192410 (2012).

[23] T. Goto, N. Kanazawa, A. Buyandalai, H. Takagi, J. Nakamura, S. Okajima, T. Hasegava, A.B. Granovsky, K. Sekiguchi, C.A. Ross, M. Inoue. Appl. Phys. Lett. 106, 132412 (2015).

[24] O. Haas, D. Dufay, S. Saez, C. Dolabdjian. IEEE Sens. J. 20, 23, 14148 (2020).

[25] А.Г. Гуревич, Г.А. Мелков. Магнитные колебания и волны. Физматлит, М. (1994). 464 с.

[26] А.С. Бугаев, Ю.В. Гуляев, П.Е. Зильберман, Ю.А. Филимонов. ФТТ. 23, 4, 2647 (1981).

[27] W. Schilz. Phil. Res. Rep. 28, 50 (1973).

Редактор Д.В. Жуманов 